\title{
Comunicação de aspectos subjetivos e intangíveis dos materiais: análise de recursos para apresentação de informações em materiotecas virtuais
}

\author{
Communicating subjective and intangible aspects of materials: an analysis \\ of the resources used to present information in virtual material libraries
}

Silvia Resende Xavier, André Carvalho Mol Silva

Materiais, materiotecas virtuais, design, comunicação

Este trabalho apresenta uma análise dos recursos utilizados para a comunicação de informações sobre aspectos subjetivos e intangíveis dos materiais listados em acervo virtual de materiotecas. São objetivos principais deste estudo a avaliação da maneira como características sensoriais e intangíveis dos materiais são comunicados em materiotecas existentes e a definição de diretrizes, pautadas por conceitos relacionados ao design da informação, para o planejamento de uma nova materioteca virtual. A metodologia contempla uma etapa de revisão de literatura acerca da importância de materiotecas e da comunicação de características sensoriais e intangíveis dos materiais no contexto de ensino e prática do design; uma etapa de análise de materiotecas existentes, guiada por conceitos da comunicação visual e design da informação; e etapas de análise e síntese dos resultados. A discussão dos resultados evidencia a efetividade de algumas abordagens adotadas para a apresentação de informações em materiotecas virtuais e destaca pontos críticos. Finalmente são definidas diretrizes para guiar o planejamento e implantação de materiotecas em meio virtual.

Materials, virtual material libraries, design, communication

This paper presents an analysis of the resources used to communicate information about subjective and intangible aspects of the materials presented in virtual material collections. The main objectives of this study are the evaluation of how sensory and intangible characteristics of materials are communicated in existing material libraries and the definition of guidelines for planning a new virtual material library, guided by concepts related to information design. The methodology includes literature review about the importance of material libraries and the communication of materials' sensory and intangible characteristics in the context of design teaching and practice; the evaluation of existing material libraries guided by concepts of visual communication and information design; and results' analysis and synthesis. The discussion of the results shows the effectiveness of some approaches adopted for the presentation of information in virtual material libraries and presents critical points. Finally, guidelines are defined for the planning and implementation of material libraries in a virtual environment.

\section{Introdução}

Em projetos de design que envolvem a concepção e fabricação de produtos, a materialização é uma atividade tão importante quanto desafiadora. Akin e Pedgley (2016) definem que a materialização é o meio pelo qual as ideias de design passam de um domínio cognitivo ou

Anais do $10^{\circ} \mathrm{CIDI}$ e $10^{\circ} \mathrm{CONGIC}$

Kelli C.A.S. Smythe, Rafael de Castro Andrade (orgs.)

Sociedade Brasileira de Design da Informação - SBDI

Curitiba | Brasil | 2021
Proceedings of the $10^{\text {th }} \mathrm{CIDI}$ and $10^{\text {th }}$ CONGIC

Kelli C.A.S. Smythe, Rafael de Castro Andrade (orgs.)

Sociedade Brasileira de Design da Informação - SBDI Curitiba | Brazil | 2021 
virtual para um domínio da realidade, da forma física. Neste processo, a seleção de materiais (SM) tem um papel central, influenciando diretamente a qualidade do resultado. Como afirmam Barauna et al. (2015) "uma característica essencial para o sucesso no desenvolvimento de produtos é a criteriosa SM com base no conhecimento sobre as capacidades e oportunidades oferecidas por todos os materiais candidatos" (p. 7). Aspectos importantes de um produto como, por exemplo, resistência, durabilidade, segurança, adequação ao uso, custo, estética e acabamento, estão intrinsecamente atrelados ao material que o compõem.

No entanto, a SM pode ser uma tarefa complexa devido à quantidade de variáveis a serem consideradas e à crescente gama de opções disponíveis. Wilkes e Miodownik (2018) mencionam que, na década de 90 , estimava-se a existência de 80.000 materiais disponíveis para a fabricação de produtos, enquanto a estimativa atual é de 160.000. Está se tornando cada vez mais difícil para os profissionais que devem materializar seus projetos, como designers, arquitetos e engenheiros, ter um conhecimento aprofundado para informar suas escolhas, em um espectro tão amplo de materiais (Wilkes \& Miodownik, 2018).

Neste contexto, iniciativas de organização e disponibilização de informações sobre materiais se difundiram em todo o mundo a partir do final dos anos de 1990 (Dantas \& Bertoldi, 2016). Entre estas iniciativas estão as materiotecas - acervos de materiais que, por meio de sistemas de classificação e catalogação, possibilitam que usuários encontrem amostras e informações do material desejado (Neves \& Pagnan, 2018). No campo educacional, as materiotecas contribuem para a formação e prática profissional em design, permitindo que estudantes e jovens profissionais tenham acesso a informações para ampliar seu repertório e seu conhecimento a respeito dos materiais e suas possibilidades de uso. Segundo Neves e Pagnan (2018), "a implantação de uma materioteca em Instituições de Ensino Superior fortalece o ensino, pesquisa e extensão, auxilia professores como ferramenta de apoio, aliando a prática à teoria" (p. 2).

$\mathrm{Na}$ Universidade Federal de Juiz de Fora (UFJF) está em fase de implantação uma materioteca com foco em design gráfico e embalagens. O projeto é parte de uma atividade de Treinamento Profissional da universidade, envolve professores e alunos do Bacharelado em Design e tem como objetivo a construção de um acervo de materiais que permita acesso e consultas em meio físico e virtual. Como parte do projeto foram realizadas pesquisas relacionadas a aspectos da atividade de SM e da construção de materiotecas, além da identificação e análise de materiotecas existentes.

O presente artigo relata os resultados de uma investigação realizada no escopo deste projeto. Para a construção de uma plataforma virtual que permita a exposição e consulta ao acervo da materioteca, um dos desafios identificados é a comunicação de um conjunto de informações relacionadas aos materiais: suas características sensoriais e estéticas, além de aspectos intangíveis, relacionados a valores associativos e emocionais (Akin \& Pedgley, 2016; Ashby \& Johnson, 2011; Barauna et al., 2015; Dantas \& Bertoldi, 2016). Em situações de acesso ao acervo físico, estas informações podem ser captadas pelo usuário da materioteca por meio de contato direto com as amostras de material. Já no contexto de apresentação virtual do acervo, estas informações devem ser traduzidas para comunicação em linguagem verbal e visual. 
Neste contexto, foi realizada uma pesquisa com objetivo geral de avaliar a maneira como aspectos sensoriais e intangíveis dos materiais são comunicados em materiotecas virtuais existentes. Os objetivos específicos deste estudo são (i) apontar a importância de materiotecas no contexto da formação em design; (ii) descrever a relevância das informações sobre aspectos sensoriais e intangíveis para a atividade de SM em projetos de design e (iii) descrever e analisar a maneira como materiotecas existentes, que possuem plataforma virtual, expõem tais informações. Espera-se, com base na análise dos resultados desta pesquisa, definir diretrizes para a apresentação de informações na plataforma virtual da Materioteca de Design da UFJF.

\section{Métodos de pesquisa}

Os métodos utilizados para o desenvolvimento desta pesquisa foram organizados em quatro fases. A primeira etapa consiste na construção da base teórica e é estruturada por meio da revisão de literatura sobre (i) a constituição de materiotecas e sua relação com o ensino e prática de design e (ii) as informações necessárias para a atividade de SM, com destaque para aquelas relacionadas a aspectos sensoriais e intangíveis dos materiais. A segunda fase da pesquisa é uma etapa exploratória, que consiste na investigação das plataformas virtuais de materiotecas existentes, visando identificar e analisar a maneira como são apresentadas as informações sobre aspectos sensoriais, estéticos e intangíveis dos materiais. Na terceira fase, os resultados obtidos na etapa exploratória são discutidos frente às informações encontradas na revisão de literatura e aos conceitos relacionados à comunicação visual e ao design de informação, visando avaliar a adequação e efetividade dos recursos utilizados para comunicação das informações em questão. Por fim, na fase conclusiva, é apresentada a compreensão geral dos resultados e a síntese do estudo.

\section{Desenvolvimento}

Nos tópicos desta seção são apresentados os resultados das etapas de revisão de literatura e da fase exploratória, que consiste na análise de materiotecas existentes.

\section{Materiotecas no contexto do ensino de design}

Como afirmam Barauna et al. (2015), a escolha de um material adequado é uma das tarefas críticas em um projeto de design e, para embasar esta seleção, é crucial ter acesso a informações sobre os materiais disponíveis para uso. Segundo os autores, alguns dificultadores para a tomada de decisão na SM são "a falta de informações de um determinado material e/ou a pouca experiência do designer" (Barauna et al., 2015, p. 5). A falta de conhecimento ou a falta de repertório pode levar designers a fazerem escolhas equivocadas ou viciadas ao definir materiais para um projeto, o que limita a possibilidade de inovação e pode gerar resultados malsucedidos. Dada a sua relevância na prática profissional, o conhecimento 
sobre materiais é também um elemento fundamental para a formação em design (Pedgley et al., 2016).

Entre os meios existentes para o estudo de materiais e apoio à SM estão as materiotecas, definidas por Wilkes e Miodownik (2018) como repositórios de conhecimento similar às bibliotecas, mas que, ao invés de livros, contém materiais e informações sobre eles. Assim como as bibliotecas, materiotecas também utilizam sistemas de classificação e catalogação de suas amostras permitindo que o usuário encontre o material desejado em meio ao universo disponível e que tenha acesso às informações a ele relacionadas (Dantas \& Bertoldi, 2016). Como afirma Silva (2005), isso permite ao projetista "ter acesso, de maneira rápida, ao universo de materiais que podem atender à sua demanda e escolher, dentre deste leque, aquele que melhor se adapte à solução desejada" (p. 34).

Laughlin (2010) identifica três tipos de materiotecas, considerando o propósito das iniciativas: as comerciais, mantidas pelo pagamento de associados e/ou patrocínio de fabricantes e fornecedores de materiais; as coleções privadas ou profissionais, geralmente montadas e mantidas por escritórios ou empresas para uso de seus funcionários; e as materiotecas institucionais (ou acadêmicas), que pertencem a instituições de ensino e servem para as necessidades dos docentes e discentes. Wilkes e Miodownik (2018) afirmam que, independentemente de sua configuração, o princípio unificador destas iniciativas é o fato de serem uma ferramenta para auxiliar profissionais e estudantes de design, arquitetura e engenharia no processo de especificação de materiais.

Virtanen et al. (2017) indicam que muitas materiotecas são estabelecidas em instituições de ensino para dar suporte à formação em design e materiais. Em levantamento feito com 17 materiotecas em países da Europa, América do Norte e Ásia, Alkin e Pedgley (2016) identificaram que 12 delas têm relação com atividade educacional. Ao tratar da importância destas bibliotecas de materiais no contexto acadêmico, Neves e Pagnan (2018) afirmam que "o sistema informacional perceptivo de seleção de materiais dentro das universidades auxilia o estudo e aprimoramento de produtos para os alunos em formação" (p. 2). Dias (2009) destaca a importância da manipulação dos materiais como forma de apoiar o conhecimento e estimular novas ideias. A autora indica que:

O desenvolvimento das percepções por meio destas atividades práticas estimula o pesquisador ou projetista a instigar o processo criativo, imaginando superfícies ou estruturas com estes materiais em seus projetos. Além disso, auxilia no processo de aprendizagem e fixação da informação para os designers em formação (Dias, 2009, p. 49).

A existência de materiotecas em universidades propicia a estudantes de design o acesso a amostras dos materiais, possibilitando que experimentem, em primeira mão, características físicas e sensoriais. No entanto, há situações em que a possibilidade de contato com as amostras é limitada, sendo necessário articular outros meios para comunicar informações sensoriais e intangíveis dos materiais. É o caso, por exemplo, das materiotecas que disponibilizam seu acervo em meio virtual, em plataformas que permitem navegação e busca. Esta presença em meio virtual permite que as materiotecas tenham um grande alcance, mas 
exige um trabalho de pesquisa e planejamento para possibilitar a adequada apresentação de todas as características e informações relevantes sobre os materiais.

\section{Desafios de comunicar aspectos sensoriais e intangíveis dos materiais}

Para tomar decisões efetivas no processo de SM, designers precisam ter informações complementares de diferentes áreas, sendo necessária uma rede complexa de conhecimento e vocabulário que envolve teoria e experimentação (Akin \& Pedgley, 2016). Informações sobre características físicas, químicas e mecânicas, relacionadas à engenharia de materiais, são baseadas em análise quantitativa e já têm parâmetros de mensuração e terminologia definidos. No entanto, este conjunto de dados técnicos é apenas um 'lado' dos materiais, sendo crucial, para o design, considerar também a maneira como percebemos e interagimos com os materiais, o que requer uma abordagem diferente para o fornecimento de informações (Akin \& Pedgley, 2016).

Barauna et al. (2015) indicam que "designers têm necessidades especiais de informações sobre valores abstratos e subjetivos dos materiais, além dos técnicos e funcionais" (p. 5). Neves e Pagnan (2018) reafirmam que, para a SM em projetos de design, são necessários dados qualitativos, relacionados à percepção sensorial e à atribuição de valor. Neste ponto, Ashby e Johnson (2011) apontam uma lacuna, expondo que não há uma sistematização dos parâmetros e definição de terminologia para informar sobre estes aspectos, assim como existe para os atributos técnicos. Há, no meio acadêmico, uma discussão importante sobre como sistematizar estas variáveis para facilitar a SM (Barauna et al., 2015), no entanto, observa-se que ainda não há um alinhamento nesta definição.

Ashby e Johnson (2011, p. 30), por exemplo, se referem a este conjunto de variáveis como "personalidade do material" e enumeram quatro tipos de atributos relacionados à essa personalidade: estéticos, de associação, percebidos e emocionais. Já autores como Pedgley et al. (2016) e Karana et al. (2015) chamam este conjunto de variáveis de "material experience" (experiência material, em tradução livre) e identificam três tipos de experiência material: estética sensorial, de significado e emocional.

Esta indefinição representa um desafio para a comunicação de informações sobre aspectos relacionados à experiência material. Em situações de apresentação virtual do acervo este desafio é aumentado pela impossibilidade de contato físico com as amostras. Como mencionado por Wilkes e Miodownik (2018), muitos aspectos dos materiais são difíceis de quantificar, sendo o contato direto com o material uma maneira de obter informações em primeira mão, por meio dos sentidos e da percepção individual. No caso de materiotecas virtuais, observa-se que é necessário usar uma combinação de recursos imagéticos e verbais para transmitir tais informações ao público.

Para embasar a investigação realizada na fase exploratória desta pesquisa, que consiste na análise da apresentação de informações sobre a experiência material em acervos virtuais existentes, foi adotada uma categorização que divide a experiência material em aspectos sensoriais e intangíveis. A caracterização das categorias, apresentada na Tabela 1, foi pautada pela revisão de literatura (Dias, 2009; Ashby \& Johnson, 2011; Pedgley et al., 2016). 
Tabela 1: Definição dos aspectos sensoriais e intangíveis dos materiais. (Fonte: adaptado de Dias, 2009)

\begin{tabular}{|c|c|c|c|}
\hline Aspecto & Caracterização & Descrição & Exemplos \\
\hline Sensorial & $\begin{array}{l}\text { objetivo; tangível; } \\
\text { palpável; estético; } \\
\text { concreto }\end{array}$ & $\begin{array}{l}\text { Pode ser quantificado e } \\
\text { classificado; seu julgamento não é } \\
\text { influenciado por sentimentos ou } \\
\text { opiniões pessoais. }\end{array}$ & $\begin{array}{l}\text { odor; acústica; dureza; } \\
\text { cor; aspereza; brilho; } \\
\text { transparência }\end{array}$ \\
\hline Intangível & $\begin{array}{l}\text { subjetivo; imaterial; } \\
\text { indireto; incorpóreo; } \\
\text { abstrato }\end{array}$ & $\begin{array}{l}\text { Difícil de medir; pertinente ou } \\
\text { característico de uma cultura ou } \\
\text { de um indivíduo. }\end{array}$ & $\begin{array}{l}\text { valor percebido; } \\
\text { significados culturais; } \\
\text { relações geográficas e } \\
\text { históricas; emoções }\end{array}$ \\
\hline
\end{tabular}

\section{Recursos utilizados para comunicar aspectos sensoriais e intangíveis dos materiais}

Para esta etapa da pesquisa, foram identificadas seis materiotecas que dispõem de plataformas online para apresentação de seu acervo. Os critérios para seleção das materiotecas foram (i) possibilidade de acesso gratuito; (ii) publicação em idioma inglês ou português; e (iii) apresentação de informações relacionadas a aspectos sensoriais e intangíveis dos materiais. Para cada uma das materiotecas, foi analisada a maneira como são apresentadas as informações sobre características sensoriais e aspectos intangíveis, tanto nas ferramentas de busca do acervo, quanto na ficha de cada material. As coleções consultadas são apresentadas na Tabela 2. Todas as materiotecas são apresentadas em páginas web que permitem, além da exibição de informações, ações de busca por palavras-chave ou navegação por meio de sub-menus e links.

Tabela 2: Materiotecas consultadas

\begin{tabular}{clll}
\hline No $^{\circ}$ & Nome & Instituição elou local & Perfil operacional \\
\hline M1 & Institute of Making & University College of London, Reino Unido & Educacional \\
M2 & Rematerialise & Kingston University London, Reino Unido & Educacional \\
M3 & Material District & Rotterdam, Holanda & Comercial \\
M4 & Materials Lab & The University of Texas at Austin, EUA & Educacional \\
M5 & Materialize & Universidade de São Paulo, Brasil & Educacional \\
M6 & Mateco & São Paulo, Brasil & Comercial \\
\hline
\end{tabular}

Primeiramente, foi estudada a maneira como o acervo é apresentado na página principal. Em todos os casos, observa-se a utilização de símbolos verbais, como substantivos e rótulos (Pettersson, 2002), organizados de maneira a configurar uma lista de opções ou como forma de indicar as classificações. Considerando as informações veiculadas, observa-se que a principal maneira de categorização dos materiais, em todas as páginas, é a classificação por famílias, como por exemplo metais, cerâmicos e polímeros. Em cinco das materiotecas investigadas há outras formas de classificação dos materiais (ex: categorização por processos 
produtivos ou por características físicas) e, entre estas, há referências a aspectos sensoriais ou intangíveis dos materiais. Estas categorias, no entanto, não são coincidentes como no caso da classificação por famílias. A Tabela 3 apresenta tópicos relacionados a aspectos sensoriais ou intangíveis encontrados nas materiotecas investigadas e as categorias gerais às quais estes tópicos estão vinculados.

Tabela 3: Categorias e tópicos relacionados a características sensoriais e intangíveis.

\begin{tabular}{|c|c|c|}
\hline $\mathbf{N}^{0}$ & Categoria & Tópicos \\
\hline M1 & Curiosidades & $\begin{array}{l}\text { 1) Destrutivo, 2) Revolucionário, 3) Cheiroso/Malcheiroso, 4) } \\
\text { Transformativo, 5) Onipresente, }(+10)^{1}\end{array}$ \\
\hline M2 & Caráter & $\begin{array}{l}\text { 1) Dobrável, 2) Colorido, 3) Elástico, 4) Leve, 5) Opaco, 6) Maleável, } \\
\text { 7) Transparente. }\end{array}$ \\
\hline M3 & - & $\begin{array}{l}\text { 1) Brilho, 2) Transparência, 3) Textura, 4) Temperatura, 5) Acústica, } \\
\text { 6) Odor, (+9). }\end{array}$ \\
\hline M4 & Propriedades & $\begin{array}{l}\text { 1) Recreativo, 2) Cheiroso/Malcheiroso, 3) Histórico, 4) Texturizado, } \\
\text { 5) Ornamental, (+320). }\end{array}$ \\
\hline M5 & $\begin{array}{l}\text { Características estético- } \\
\text { sensoriais }\end{array}$ & $\begin{array}{l}\text { 1) Texturas, 2) Toque macio/duro, 3) Toque flexível/rígido, 4) Toque } \\
\text { quente/frio, 5) Toque antiaderente/antiderrapante, 6) Brilho, 7) } \\
\text { Transparência, 8) Reflexão. }\end{array}$ \\
\hline
\end{tabular}

A página web de M6 não apresenta formas de classificação relacionadas a aspectos sensoriais e intangíveis dos materiais. No caso desta materioteca, está disponível na página principal somente a categorização por família, outras classificações ou características aparecem apenas na página de apresentação de cada material. Ao analisar as páginas individuais de apresentação dos materiais observa-se um conjunto de recursos visuais e textuais que são usados para comunicar o máximo de informações do material, incluindo aspectos sensoriais e intangíveis. A Figura 1 apresenta A) um exemplo de página principal, evidenciando o uso de uma lista de termos à esquerda, como uma ferramenta de classificação e busca, e aplicação de termos como tags sobre as imagens; e B) um exemplo de página de apresentação individual de um material, expondo imagens na parte superior, seguidas de texto e lista de termos na parte inferior.

\footnotetext{
${ }^{1}$ Quando há tópicos variados na categoria, foram enumerados somente aqueles relacionados às características sensoriais e intangíveis dos materiais, sendo a quantidade de tópicos restante expressa entre parênteses, ao final.
} 
Figura 1: A) Página principal e B) página de apresentação individual de material de M3. (Fonte: adaptado de https://materialdistrict.com/material)
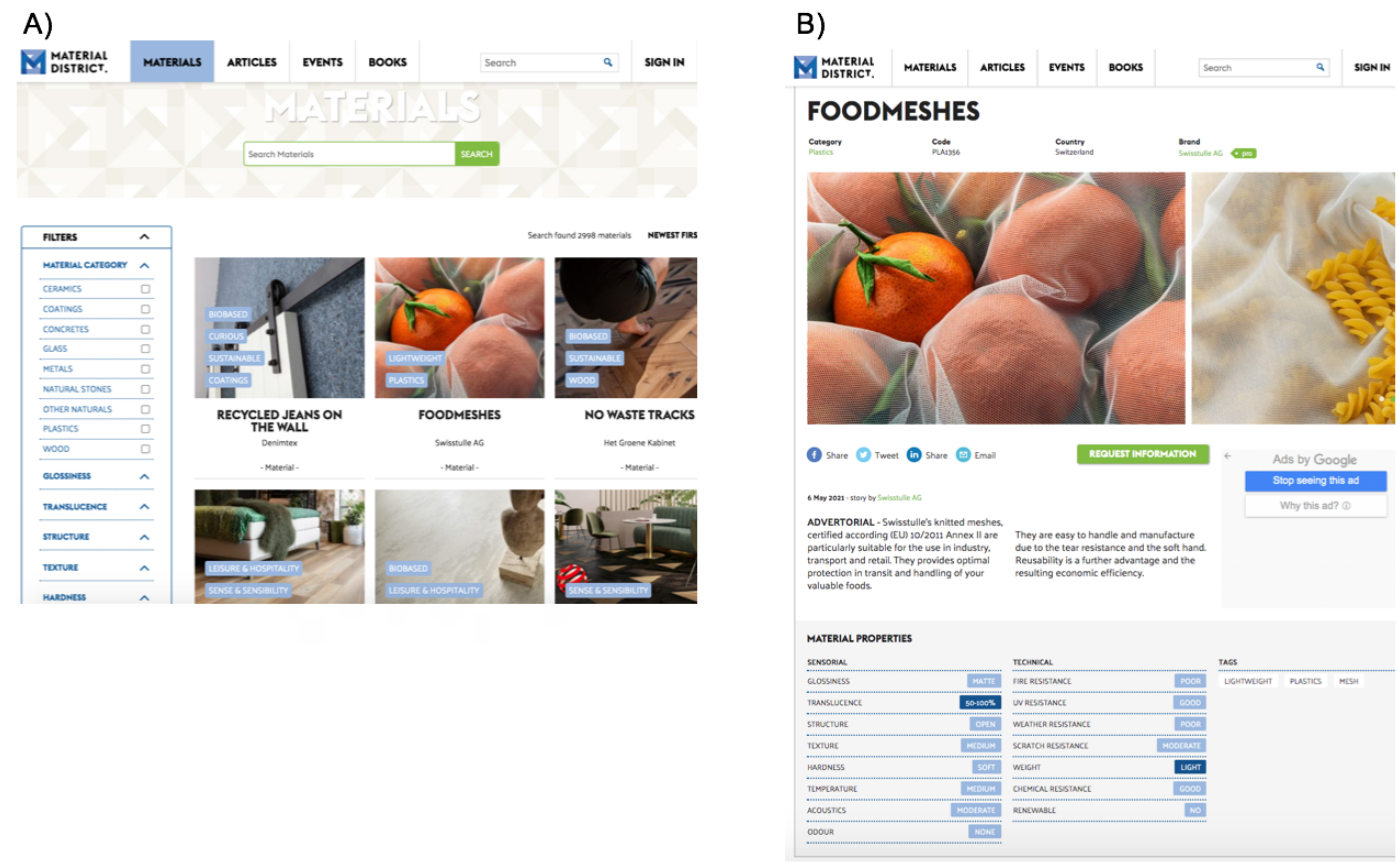

Considerando a comunicação de informações relacionadas especificamente aos aspectos sensoriais e intangíveis, alguns recursos comumente utilizados foram: (i) fotos do material sendo manipulado; (ii) fotos de detalhes; (iii) apresentação de termos ou expressões. Um destaque foi a materioteca M2, que não apresenta palavras para descrição das características, mas que, em alguns casos, dispõe de vídeo do material sendo manipulado, evidenciando assim aspectos físicos e sensoriais. As Figuras 2 a 4 apresentam exemplos de imagens de análise utilizadas para este estudo, produzidas com a montagem de imagens printscreen das páginas web das materiotecas e marcações, em linha pontilhada azul, para destacar os recursos utilizados na comunicação de aspectos sensoriais e intangíveis dos materiais.

Figura 2: Informações destacadas em M1 (Fonte: adaptado de https://www.instituteofmaking.org.uk/materials-library/material/aerogel)

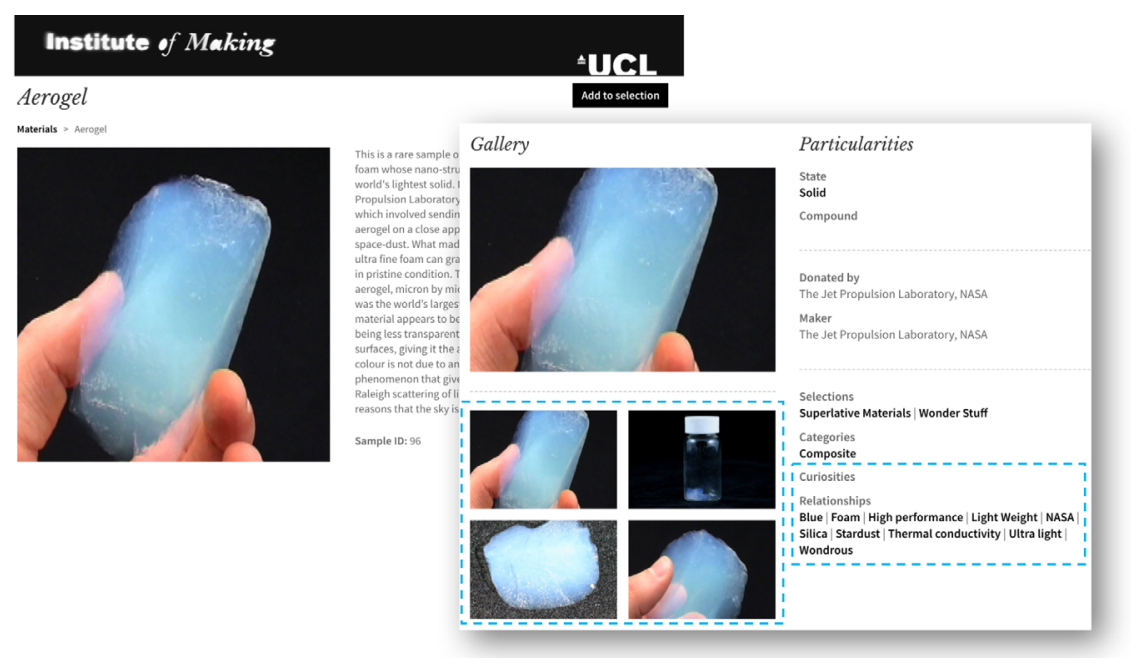


Figura 3: Informações destacadas em M2 (Fonte: adaptado de https://research.kingston.ac.uk/rematerialise/html_and_flash/index-type-vegetable.htm)

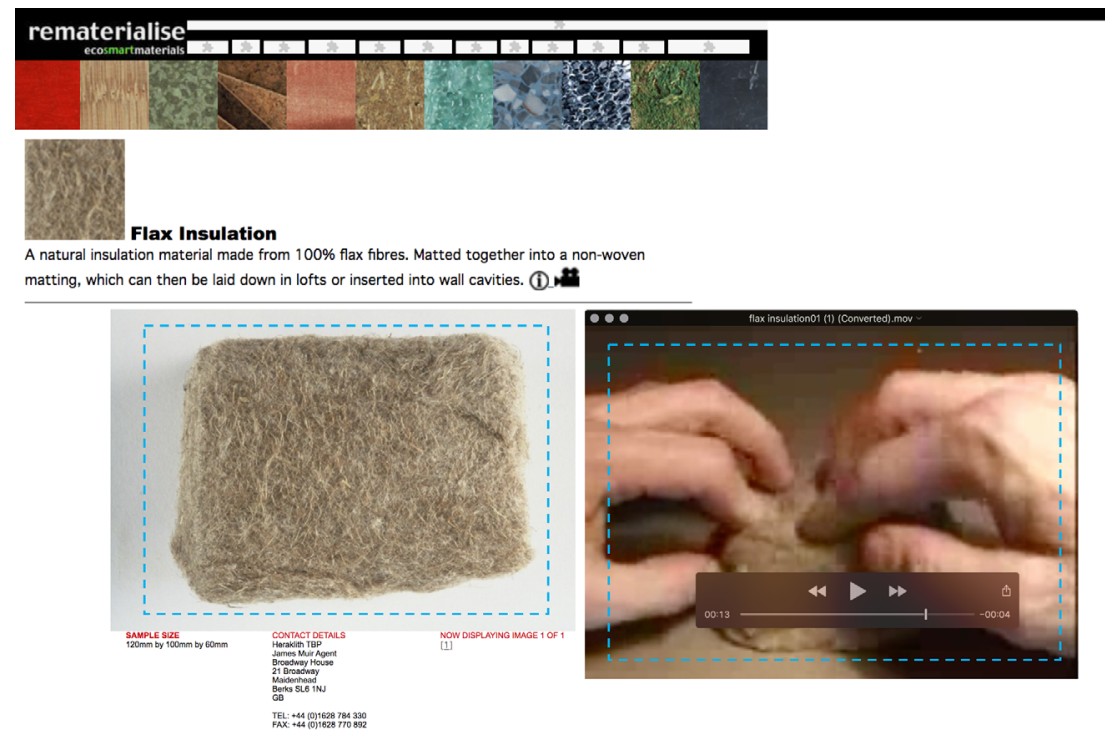

Figura 4: Informações destacadas em M3 (Fonte: adaptado de https://materialdistrict.com/material/coquim-coconut-fibre/)

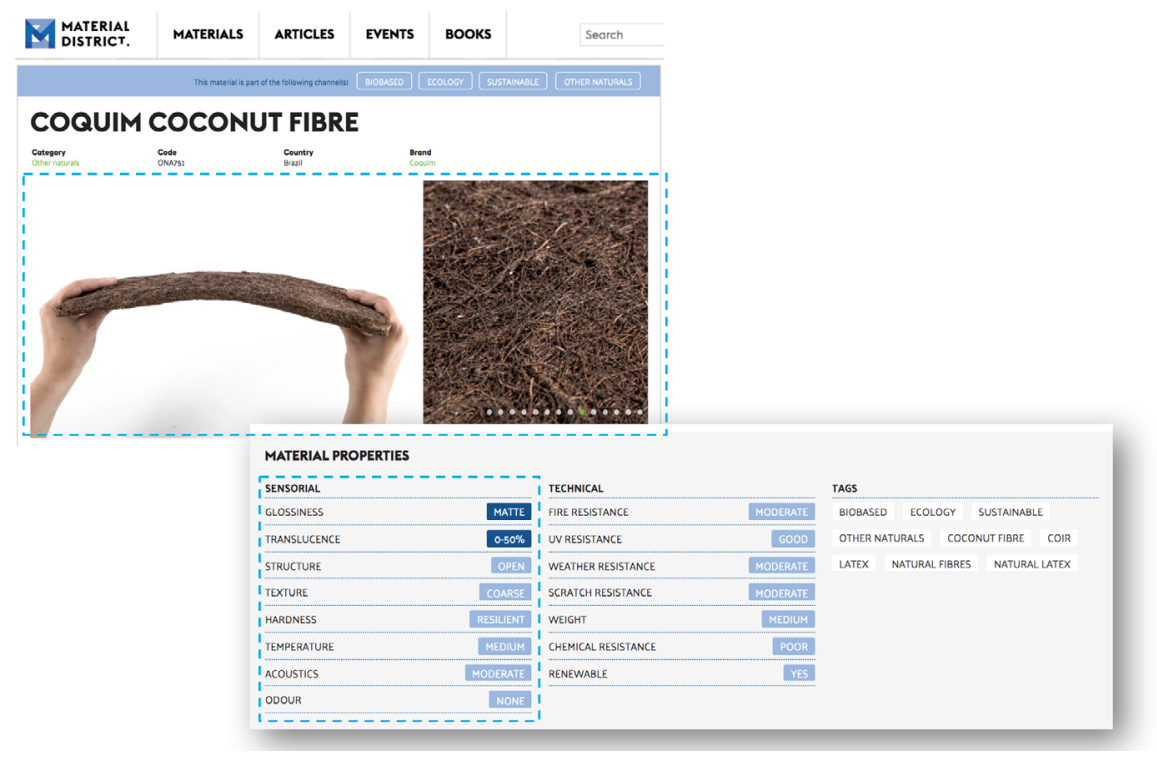

Com relação à organização das informações que são expressas em termos ou expressões curtas, observa-se que M1 e M4 não têm uma padronização de tópicos para apresentá-las, registrando quantidades e variedades diferentes de dados para cada material, como ilustrado na Figura 5. Já M3, M5 e M6 apresentam uma estrutura de tópicos, mostrando, consistentemente, informações sobre os mesmos tópicos para cada material, como mostra a Figura 6. 
Figura 5: Exemplo de apresentação não padronizada de informações em M1 (Fonte: adaptado de https://www.instituteofmaking.org.uk/materials-library)

$\begin{array}{lll}\text { Aerogel } & \text { Natural Rubber } & \text { Glass Tuning Fork } \\ \text { Selections } & \text { Selections } & \text { Selections } \\ \text { Superlative Materials } \mid \text { Wonder Stuff } & \text { Categories } & \text { Sound } \\ \text { Categories } & \text { Polymer } & \text { Categories } \\ \text { Composite } & \text { Curiosities } & \text { Glass } \\ \text { Curiosities } & \text { Smelly } & \text { Curiosities } \\ \text { Relationships } & \text { Relationships } & \text { Damaged } \\ \text { Blue } \mid \text { Foam } \mid \text { High performance } \mid \text { Light Weight } \mid \text { NASA } \mid \text { Silicaa } \mid & \text { Elastic| Electrostatic| Isoprene } \mid \text { Latex } \mid & \text { Relationships } \\ \text { Stardust } \mid \text { Thermal conductivity } \mid \text { Ultra light } \mid \text { Wondrous } & \text { Polyisoprene } \mid \text { Stretchy } \mid \text { Tree } & \text { Acoustic } \mid \text { Glass } \mid \text { Sound of Materials } \mid \text { Tuning Fork }\end{array}$

Figura 6: Exemplo de apresentação padronizada de informações em M3 (Fonte: adaptado de https://materialdistrict.com/)

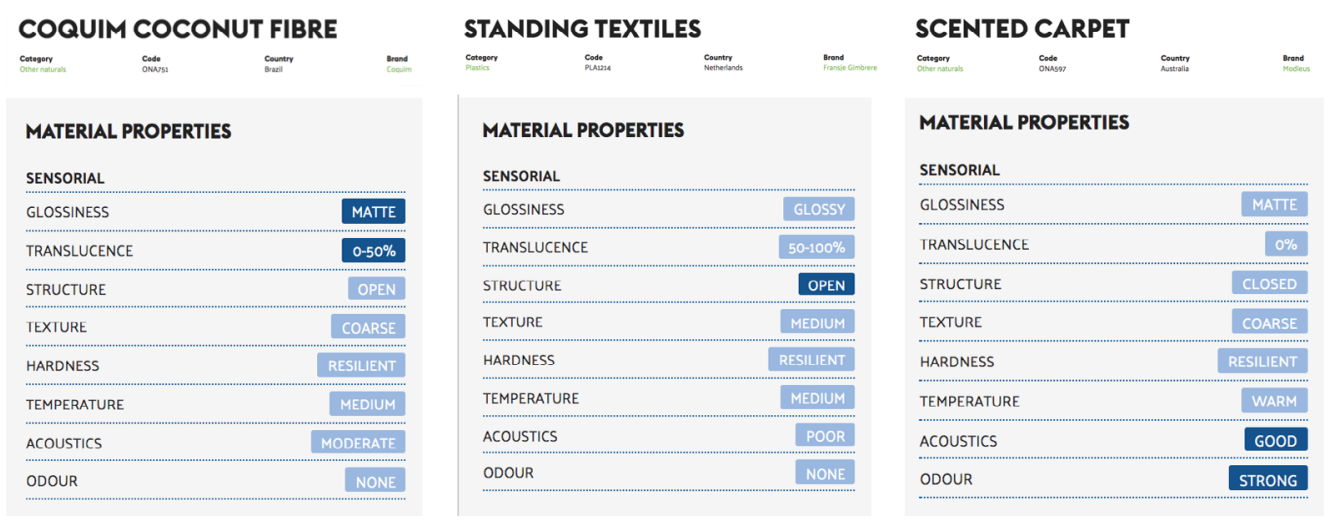

\section{Discussão de resultados}

Com base na análise das materiotecas existentes, é possível destacar pontos importantes, relacionados ao design de informação, que devem ser considerados na apresentação de uma materioteca virtual e, em especial, na comunicação de aspectos sensoriais e intangíveis dos materiais. Um ponto de destaque é o uso de website como plataforma para a apresentação da materioteca, permitindo a exposição de diversos elementos textuais e visuais, bem como a interação com o usuário.

Sobre estas interfaces em ambiente virtual, Frascara (2004) menciona que devem apresentar claramente a organização hierárquica de seu conteúdo e as ferramentas de navegação. Já Portugal et al. (2014) destacam a importância de repetições de elementos de design para trazer consistência e credibilidade para o usuário ao navegar em ambientes de hipermídia. Segundo os autores, um bom sistema utiliza "uma família tipográfica, o mesmo estilo em todos os ícones e uma hierarquia consistente de informações por meio de cores ou de outros elementos de design" (Portugal et al., 2014, p. 4). Neste sentido, considera-se que as apresentações virtuais das materiotecas investigadas, de maneira geral, cumprem estes parâmetros, apresentando consistência visual, boa organização da informação e ferramentas para navegabilidade.

A respeito das representações visuais utilizadas para comunicação de aspectos sensoriais e intangíveis dos materiais, destacou-se a apresentação de múltiplas fotografias mostrando o 
material em diferentes situações, além da apresentação de vídeo em alguns casos. Estas imagens realistas são especialmente importantes para a comunicação de aspectos sensoriais e intangíveis dos materiais pois possibilitam uma compreensão visual de algumas características, sem a necessidade de "tradução" para a linguagem verbal, que poderia retardar ou comprometer o fluxo de entendimento. Pettersson (2002) afirma que informações visuais são percebidas mais rápida e prontamente do que informações textuais, descrevendo que levamos aproximadamente 2 ou 3 segundos para reconhecer o conteúdo de uma imagem, enquanto demoramos 20 ou 30 segundos para ler uma descrição verbal da mesma imagem. Ademais, as imagens podem provocar sentimentos e emoções, além do entendimento racional. Como afirma Lester (2020), mensagens visuais estimulam resposta intelectual e emocional, elas nos fazem sentir tanto quanto nos fazem pensar. Esta reação emocional é parte importante do processo de transmissão de informações sobre o material, especialmente para o conhecimento de aspectos intangíveis.

Ao analisar as representações verbais utilizadas, observa-se que palavras únicas ou expressões curtas foram empregadas com frequência para definir as categorias de informação ou para denominar os tópicos nestas categorias. No caso da apresentação de aspectos sensoriais e intangíveis dos materiais, os termos eram também utilizadas para definir, qualitativamente, aquela característica. Em M2, por exemplo, na descrição das propriedades do material, há a categoria "sensorial" e o tópico "dureza", que pode ser definido como "macio", "resiliente" ou "duro". Pettersson (2002, p. 84) indica que o uso de termos ou expressões técnicas já consolidadas permite a comunicação de conceitos ou ideias de maneira concisa, sem a necessidade de uma explicação exaustiva.

Considerando ainda o emprego de termos ou expressões para a comunicação de características dos materiais, destaca-se a importância da sistematização, apresentando consistentemente o mesmo conjunto de tópicos para todos os materiais, como foi observado em M3, M5 e M6. A falta de padronização na apresentação dos tópicos, como aconteceu em M1 e M4, pode deixar lacunas no entendimento. Por exemplo: ao acessar a página de apresentação de um material em M1 temos o tópico "smell", caracterizando o material como tendo um cheiro forte. No entanto, ao acessar a página de um outro material, não temos nenhum tópico sobre o cheiro, ficando indefinido se é um material inodoro, com odor suave, ou se essa informação foi apenas ignorada.

Neste ponto, é importante também considerar a falta de definição de vocabulário e parâmetros de mensuração para expressar aspectos sensoriais e subjetivos, o que pode ser um dificultador para o planejamento da comunicação. Pedgley et al. (2016) registram que falta desenvolvimento de vocabulário e conceitos que podem ser usados para descrever as experiências materiais reais e pretendidas. Já Barauna et al. (2015) destacam que, neste contexto, "a padronização dos termos facilitaria a obtenção das informações" (p. 6).

Por fim, considerando as ferramentas de busca disponíveis e a maneira de organizar as informações, destaca-se a possibilidade apresentada por quase todas as materiotecas (com exceção de M6) de visualizar categorizações ou realizar buscas com base em aspectos 
diversos, não apenas com base na classificação de famílias. Esta possibilidade vai ao encontro das necessidades dos designers, como destaca Pedgley et al. (2016) "estudantes e profissionais de design ficam mais confortáveis começando investigações para escolha de material de uma perspectiva de caracterização sensorial-expressiva do que começando com requerimentos técnicos" (p. 614, tradução livre).

Finalmente, com base nas análises feitas anteriormente, foi possível definir diretrizes para a apresentação de materiotecas em meio virtual, considerando princípios do design da informação e tendo como foco a comunicação de aspectos sensoriais e intangíveis:

- a utilização de website como plataforma de apresentação do acervo é adequada e funcional, respeitando parâmetros de organização da informação, navegabilidade e consistência visual;

- o emprego de fotografias e/ou vídeos é indicado para comunicar aspectos físicos e sensoriais de maneira clara e direta, além de suscitar sentimentos e emoções que podem contribuir para o conhecimento de aspectos intangíveis do material;

- o uso de representações verbais também é efetivo, principalmente no formato de palavras únicas ou expressões curtas, destacando-se a importância de padronizar o conjunto de informações apresentadas nas fichas dos materiais;

- a possibilidade de busca com base em categorizações diversas, relacionadas a aspectos sensoriais e intangíveis deve ser considerada, além da tradicional categorização por famílias ou por parâmetros técnicos.

\section{Conclusões}

Este estudo apresentou uma revisão, realizada por meio de pesquisa bibliográfica, da caracterização de materiotecas e sua relevância no contexto de ensino de design, focando na discussão acerca da importância das características sensoriais e intangíveis dos materiais e dos desafios relacionados à comunicação destas características em situações de apresentação virtual do acervo. Uma investigação sobre a comunicação destas características foi realizada por meio de uma análise de materiotecas existentes, pautada por conceitos da comunicação visual e do design da informação.

A discussão de resultados evidencia a efetividade de algumas abordagens adotadas para a apresentação das informações em materiotecas virtuais, como (i) o uso de websites com boa organização hierárquica da informação, disponibilidade de ferramentas de navegação e consistência visual; (ii) a exibição de fotografias diversas e/ou vídeo do material; (iii) o uso de representações verbais, principalmente no formato de palavras ou expressões curtas; (iv) a possibilidade de navegação por categorizações diversas, não apenas a tradicional divisão de famílias de materiais. Como ponto crítico, destaca-se a falta de padronização do conjunto de informações apresentados para cada material, em alguns casos, o que pode deixar lacunas no entendimento. Com base nas análises realizadas, foram definidas diretrizes para guiar 0 planejamento e implantação de materiotecas em meio virtual. 


\section{Agradecimento}

Os autores agradecem ao PROQUALI da UFJF pela bolsa de capacitação.

\section{Referências}

Akin, F., \& Pedgley O. (2016). Sample libraries to expedite materials experience for design: A survey of global provision. Materials \& Design, 90, 1207-1217. https://doi.org/10.1016/j.matdes.2015.04.045

Ashby, M. F., \& Johnson, K. (2011). Materiais e design: Arte e ciência da seleção de materiais no design de produto. Rio de Janeiro: Elsevier.

Barauna, D., Razera, D. L., \& Heemann A. (2015). Seleção de Materiais no Design: Informações Necessárias ao Designer na Tomada de Decisão para a Conceituação do Produto. Design \& Tecnologia, 5(10), 1-9. https://doi.org/10.23972/det2015iss10pp1-9

Dantas, D., \& Bertoldi, C. A. (2016). Cataloguing and indexing system for samples of materials related to design projects for the use in materials collections. DAT Journal, 1(2), 62-75. https://doi.org/10.29147/2526-1789.DAT.2016v1i2p62-75

Dias, M. R. A. C. (2009). Percepção dos materiais pelos usuários: modelo de avaliação Permatus. 2009, 291 f. Tese (Doutorado em Engenharia e Gestão do Conhecimento) Universidade Federal de Santa Catarina, Florianópolis.

Frascara, J. (2004). Communication design: principles, methods and practice. Nova lorque: Allworth Press.

Institute of Making. (n.d.). Materials Library. Disponivel em https://www.instituteofmaking.org.uk/materials-library

Karana, E., Pedgley, O., \& Rognoli, V. (2015). On Materials Experience. Design Issues 3 (3), 16-27. https://doi.org/10.1162/DESI_a_00335

Laughlin, Z. (2010). Beyond the Swatch: How Can the Science of Materials Be Represented by the Materials Themselves in a Materials Library? [Tese de doutorado]. Departamento de Engenharia. King 's College London, University of London, Reino Unido. https://doi.org/10.13140/RG.2.2.16034.94405

Lester, P. M. (2020). Visual Communication: Images with Messages. $8^{a}$ edição. Dallas: Lex Publishing.

Mateco. (n.d.). Materiais. Disponível em https://mateco.wordpress.com/produtos/

Material District. (n.d.). Materials. Disponível em https://materialdistrict.com/material/

Materialize. (n.d.). Pesquisa no acervo. Disponível em http://www.materialize.fau.usp.br/index.php/home

Materials Lab. (n.d.). Materials Lab. Disponível em https://materials.soa.utexas.edu/search/index/

Neves, H. L., \& Pagnan, A. S. (2018). A importância da materioteca como apoio ao ensino de design. Colóquio Internacional de Design 2017 - Blucher Design Proceedings, 4, 16-28. http://dx.doi.org/ 10.5151/cid2017-02 
Pedgley, O., Rognoli, V. \& Karana, E. (2016). Materials experience as a foundation for materials and design education. Int J Technol Des Educ, 26, 613-630. https://doi.org/10.1007/s10798015-9327-y

Pettersson, R. (2002). Information Design: an introduction. Amsterdam: John Benjamins Publishing.

Portugal, C., Pereira, M., Corrêa, M., \& Lasmar, R. (2014). Design da informação em sistema digital para mapeamento dos sítios de endometriose. Anais do $11^{\circ}$ Congresso Brasileiro de Pesquisa e Desenvolvimento em Design - Blucher Design Proceedings, 1(4). São Paulo: Blucher. https://doi.org/2014.10.5151/designpro-ped-00230

Rematerialise. (n.d.). Eco Smart Materials. Disponível em https://research.kingston.ac.uk/rematerialise/html_and_flash/searchwelcome.htm

Silva, E. S. A. (2005). Um sistema informacional e perceptivo de seleção de materiais com enfoque no design de calçados. [Dissertação de mestrado]. Escola de Engenharia. Universidade Federal do Rio Grande do Sul, Brasil.

Virtanen, M., Manskinen, K., \& Eerola, S. (2017). Circular Material Library. An Innovative Tool to Design Circular Economy. The Design Journal, 20(1), 1611-1619. https://doi.org/10.1080/14606925.2017.1352685

Wilkes, S. E., \& Miodownik, M. A. (2018). Materials library collections as tools for interdisciplinary research. Interdisciplinary Science Reviews, 43(1), 3-23.

https://doi.org/10.23972/det2015iss10pp1-9

\section{Sobre os autores}

André Carvalho Mol Silva, Me., UFJF, Brasil <andre.mol@design.ufjf.br> Silvia Resende Xavier, Ma., UFJF, Brasil <silvia.xavier@design.ufjf.br> 